\title{
Duality transformation and conformal equivalent scalar-tensor theories
}

\author{
Gabriele Gionti, S.J.1,2,* and Andronikos Paliathanasis ${ }^{3,4, \dagger}$ \\ ${ }^{1}$ Specola Vaticana, V-00120 Vatican City, Vatican City State, \\ Vatican Observatory Research Group, Steward Observatory, \\ The University Of Arizona, 933 North Cherry Avenue, Tucson, Arizona 85721, USA \\ ${ }^{2}$ INFN, Laboratori Nazionali di Frascati, Via E. Fermi 40, 00044 Frascati, Italy \\ ${ }^{3}$ Instituto de Ciencias Fúsicas y Matemáticas, Universidad Austral de Chile, Valdivia, Chile \\ ${ }^{4}$ Institute of Systems Science, Durban University of Technology, \\ PO Box 1334, Durban 4000, Republic of South Africa
}

\begin{abstract}
We deal with the duality symmetry of the Dilaton field in cosmology and specifically with the socalled Gasperini-Veneziano duality transformation. In particular, we determine two conformal equivalent theories to the Dilaton field, and we show that under conformal transformations GasperiniVeneziano duality symmetry does not survive. Moreover, we show that those theories share a common conservation law, of Noetherian kind, while the symmetry vector which generates the conservation law is an isometry only for the Dilaton field. Finally, we show that the Lagrangian of the Dilaton field is equivalent with that of the two-dimensional "hyperbolic oscillator" in a Lorentzian space whose $O(d, d)$ invariance is transformed to the Gasperini-Veneziano duality invariance in the original coordinates.
\end{abstract}

PACS numbers: 98.80.-k, 95.35.+d, 95.36.+x

Keywords: Cosmology; Gasperini-Veneziano Duality; Coformal equivalnece; Scalar tensor; $f(R)$-gravity

\section{INTRODUCTION}

Theories of gravity which extend General Relativity have drawn the attention of the scientific society in that last decade because they provide geometric methods to solve the problem of the late-time acceleration of the universe without the necessity to consider dark energy, for instance see [1-6]. On the other hand, an important property of the two-dimensional conformal field theory is the duality symmetry which has important consequences in cosmology [9-15]. The duality symmetry is characterized by the invariance of the action integral, therefore the corresponding Euler-Lagrange equations, under this transformation, remain the same. Usually, in String Theory, when the "radius" $\mathcal{R}$ of the compactified geometry changes such as $\overline{\mathcal{R}} \rightarrow \mathcal{R}^{-1}$. Duality transformation is a discrete transformation and an isometry should exist for the underlying manifold $[7,8]$.

If one considers the tree level effective action of gravity minimally coupled with the Dilaton field, derived by imposing, at one loop, the conformal invariance of the sigma model at quantum level $[7,8]$, then they get

$$
S=\int d^{4} x \sqrt{-G} e^{-2 \phi}\left(R-4 G^{\mu \nu} \phi_{; \mu} \phi_{; \nu}+\Lambda-\frac{1}{12} H_{\mu \nu \rho} H^{\mu \nu \rho}\right) .
$$

Here $\Lambda$ is the cosmological constant, $\phi$ the Dilaton field, $H_{\mu \nu \rho}$ is expressed in terms of $B_{\mu \nu}$ as $H_{\mu \nu \rho}=\partial_{\mu} B_{\nu \rho}+$ cyclic permutations. In case $G, B$ and $\phi$ are functions of the time only $[16,17]$, one can prove that

$$
G=\left(\begin{array}{cc}
-1 & 0 \\
0 & G(t)
\end{array}\right), B=\left(\begin{array}{cc}
0 & 0 \\
0 & B(t)
\end{array}\right)
$$

It has been shown in [18] that introducing a $2 d \times 2 d$ matrix and a field $\Psi$

$$
M=\left(\begin{array}{cc}
G^{-1} & -G^{-1} B \\
B G^{-1} & G-B G^{-1} B
\end{array}\right), \Psi=2 \phi-\ln \sqrt{\operatorname{det} G}
$$

\footnotetext{
*Electronic address: ggionti@specola.va
}

$\dagger$ Electronic address: anpaliat@phys.uoa.gr 
with these positions the previous action (1) becomes

$$
S=\int d t e^{-\Psi}\left(\Lambda+(\dot{\Psi})^{2}+\frac{1}{8} \operatorname{Tr}[\dot{M} \eta \dot{M} \eta]\right)
$$

in which $\eta_{\sigma \lambda}$ is the $O(d, d)$ off diagonal metric defined as

$$
\eta_{\sigma \lambda}=\left(\begin{array}{ll}
0 & 1 \\
1 & 0
\end{array}\right)
$$

Furthermore, it is straightforward to see that action (4) is $O(d, d)$ invariant, that is, $\Psi \mapsto \Psi, M \mapsto \Omega^{T} M \Omega$, where $\Omega^{T} \eta \Omega=\eta$. In the case $B=0$ and $\Omega=\eta$, one gets the "reflection" of the $O(d, d)$ group which is formulated as follows

$$
G(t) \mapsto G(t)^{-1} \quad, \phi \mapsto \phi-\ln \sqrt{\operatorname{det} G}
$$

so that considering a spatially flat Friedmann-Lemaître-Robertson-Walker metric (FLRW) with scale factor $a(t)$, and $\sqrt{-g}=a^{3}$, the $O(d, d)$ transformation becomes the Gasperini-Veneziano duality transformation $a \mapsto a^{-1}$ and $\phi \mapsto$ $\phi-3 a$.

Indeed, the Gasperini-Veneziano duality transformation maps solutions into solutions, and being generated, in general, by a continuous group, $O(d, d)$ its is associated with an isometry of the Lagrangian, for a review see [19]. This means that there exists a Vector field $X$ such that the Lie derivative $L_{X}$ of the Lagrangian $\mathcal{L}$ is zero $L_{X} \mathcal{L}=$ 0 , which means that $X$ is an isometry for the Lagrangian. That is an important property for the existence of a duality transformation and is used later to explain how duality symmetry is lost under conformal transformations, for conformal equivalent theories.

Recently in [21] a similar transformation to that of the Gasperini-Veneziano duality transformation [20] was studied and it's connection with point symmetries in the case of the modified gravitational fourth-order theory, the $f(R)$ gravity, while a study on the relation between continuous and discrete symmetries in gravitational theories recently performed in $[22]$.

In this letter we revise the problem and in our approach we consider the property, that scalar-tensor theories and $f(R)$-gravity are conformal equivalent. We show that because of this constraint those conformal equivalent theories share a common invariant transformation for the Lagrangian in which only for the Dilaton field, that transformation reduce to the duality Gasperini-Veneziano duality transformation.

\section{CONFORMAL EQUIVALENT THEORIES AND SYMMETRIES}

For the convenience of the reader we briefly discuss the conformal equivalence of scalar-tensor theories. Let $\varphi$ be a minimally coupled scalar field in which the field equations are given by the variation of the action Integral

$$
S_{\varphi}=\int d^{4} x \sqrt{-g}\left(F(\varphi) R(g)-\frac{1}{2} g^{\mu \nu} \varphi_{; \mu} \varphi_{; \nu}+V(\varphi)\right)
$$

where $g_{\mu \nu}$ is the metric tensor for a four-dimensional Riemannian space of Lorentzian signature, and Ricciscalar $R(g)$.

Consider the conformal equivalent metric $g_{\mu \nu}=N^{2}\left(x^{k}\right) \gamma_{\mu \nu}$, where now the Ricciscalars $R(\gamma)$, and $R(g)$ are related as follows $[23]$

$$
R(g)=N^{-2} R(\gamma)-6 N^{-3} \gamma^{\mu \nu} N_{; \mu \nu}
$$

By replacing (8) in (7) it follows

$$
S_{\varphi}=\int d^{4} x \sqrt{-\gamma}\left(F(\varphi) N^{2} R(\gamma)-\frac{1}{2} N^{2} \gamma^{\mu \nu} \varphi_{; \mu} \varphi_{; \nu}+N^{4} V(\varphi)-6 F(\varphi) N \gamma^{\mu \nu} N_{; \mu \nu}\right)
$$

Integration by parts in the last term of expression (9) gives

$$
A_{0}=\int d^{4} x \sqrt{-\gamma}\left(6 N F_{, \varphi} \gamma^{\mu \nu} N_{; \mu} \phi_{; \nu}+6 F(\varphi) \gamma^{\mu \nu} N_{; \mu} N_{; \nu}\right)
$$


Function $N\left(x^{k}\right)$ is not a real degree of freedom and without loss of generality can be neglected by assuming that $N\left(x^{k}\right)=N\left(\varphi\left(x^{k}\right)\right)$. Hence with that consideration the Action Integral (9) becomes

$$
S_{\varphi}=\int d^{4} x \sqrt{-\gamma}\left(F(\varphi) N^{2} R(\gamma)-\frac{1}{2} W(\varphi) \gamma^{\mu \nu} \varphi_{; \mu} \varphi_{; \nu}+\hat{V}(\varphi)\right)
$$

where

$$
W(\varphi)=-2\left(-\frac{1}{2} N^{2}+6 N F_{, \varphi} N_{, \varphi}+6 F(\varphi)\left(N_{, \varphi}\right)^{2}\right), \hat{F}(\varphi)=F(\varphi) N^{2}, \text { and, } \hat{V}(\varphi)=N^{4} V(\varphi)
$$

The action integrals, (7), and (11), are different but they describe two conformal equivalent scalar-tensor theories. Moreover, in order to make clear that actions (7), and (11) describe different fields, we define the new field $\Phi$ such that $\Phi=\int \sqrt{W(\varphi)} d \varphi$, in which (11) takes the simplest form [1]

$$
S_{\Phi}=\int d^{4} x \sqrt{-\gamma}\left(\hat{F}(\Phi) R-\frac{1}{2} \gamma^{\mu \nu} \Phi_{; \mu} \Phi_{; \nu}+\hat{V}(\Phi)\right)
$$

It is important to mention here that when $\hat{F}(\Phi)=$ const., that is $F(\varphi) N(\varphi)^{2}=$ const., the action integral (13) describes a minimally coupled scalar field model.

Consider now the Dilaton field with action integral (1) and $H_{\mu \nu \rho}=0$, that is,

$$
S_{(\text {Dilaton })}=\int d^{4} x \sqrt{-g} e^{-2 \phi}\left(R-4 g^{\mu \nu} \phi_{; \mu} \phi_{; \nu}+\Lambda\right)
$$

which without loss of generality can be written in the form of the Action (7) with $F(\varphi)=\frac{1}{8} \varphi^{2}$, and $V(\phi)=\frac{\Lambda}{8} \varphi^{2}$, while the two fields $\phi$, and $\varphi$ are related as $2 \sqrt{2} e^{-\phi}=\varphi$. Consequently, from the above formulas we find that the minimally coupled scalar field equivalent Action is

$$
S_{A}=\int d^{4} x \sqrt{-\gamma}\left(R-\frac{1}{2} \gamma^{\mu \nu} \Phi_{; \mu} \Phi_{; \nu}+\hat{\Lambda} e^{-\lambda \Phi}\right)
$$

in which $\hat{\Lambda}=64 \Lambda$ and $\lambda=\frac{1}{\sqrt{5}}$. However, from our discussion now it is clear that the later action is not unique and there exists a family of conformal equivalent actions with the Dilaton field (14). For instance if we perform the (second) conformal transformation $\gamma_{\mu \nu}=e^{\frac{\sqrt{3}}{3} \Phi} \kappa_{\mu \nu}, \Phi=\sqrt{3} \ln \sigma$, in (15), we find the conformal equivalent action

$$
S_{B}=\int d^{4} x \sqrt{-\gamma}\left(\sigma R+\hat{\Lambda} \sigma^{\mu}\right), \mu=\left(2-\frac{\sqrt{3}}{\sqrt{5}}\right)
$$

which is the O'Hanlon action with a power-law potential $V(\sigma) \simeq \sigma^{\mu}[25]$.

It is well-known that O'Hanlon theory is equivalent with $f(R)$-gravity [24] with the use of Lagrange multiplier $[26,27]$. Specifically, it is an alternative way to write the action of $f(R)$-gravity, as a special case of Hordenski theory [28]. The potential of the O'Hanlon theory and the $f(R)$-gravity are related by the Clairaut equation $f_{, R}(R) R-$ $f(R)=V\left(f_{, R}\right)$, where for the power-law potential in (16) the $f(R)$ equivalent theory is derived to be the power-law $f(R)$ model.

$$
f(R) \simeq R^{\frac{\mu}{\mu-1}}, \mu=\left(2-\frac{\sqrt{3}}{\sqrt{5}}\right) .
$$

Now consider the underlying space to be that of FLRW geometry with zero spatial curvature, that is

$$
d s^{2}=-N^{2}(t) d t^{2}+a^{2}(t)\left(d x^{2}+d y^{2}+d z^{2}\right)
$$

where $a(t)$ is the scale factor and $N(t)$ is the lapse function. We continue by assuming that the matter source inherits the spacetime symmetries. 
For the three conformal equivalent actions ${ }^{1},(14),(15)$, and (16) for the Dilaton field $\varphi$, the minimally coupled field $\Phi$, and the $f(R)$ gravity (field $\sigma$ ), respectively; the field equations in the spatially flat FLRW background space (18) are described by the following point-like Lagrangians

$$
\begin{gathered}
L_{\varphi}(a, \dot{a}, \varphi, \dot{\varphi})=\frac{1}{N}\left(6 a \varphi^{2} \dot{a}^{2}+12 a^{2} \varphi \dot{a} \dot{\varphi}-4 a^{3} \dot{\varphi}^{2}\right)-\Lambda N a^{3} \varphi^{2}, \\
L_{\Phi}(a, \dot{a}, \Phi, \dot{\Phi})=\frac{1}{N}\left(3 a \dot{a}^{2}-\frac{1}{2} a^{3} \dot{\Phi}^{2}\right)+\hat{\Lambda} N e^{-\lambda \Phi},
\end{gathered}
$$

and

$$
L_{\sigma}(a, \dot{a}, \sigma, \dot{\sigma})=\frac{1}{N}\left(6 a \sigma \dot{a}^{2}+6 a^{2} \dot{a} \dot{\sigma}\right)+\hat{\Lambda} N \sigma^{\mu}
$$

Those three point-like Lagrangians are singular, and are conformally related because of the constraint condition $\mathcal{H} \equiv \frac{\partial L}{\partial N}=0$. Without loss of generality in the following we set $N(t)=1$.

We observe that only Dilaton's Lagrangian (19) is invariant under the Gasperini-Veneziano duality transformation, which on the coordinates $\{a, \varphi\}$ is $a \rightarrow a^{-1}, \varphi \rightarrow \varphi a^{3}$. The three point-like Lagrangians share another property, that is, all admit linear in the momentum conservation laws which are generated by the same conformal killing vector field of the two dimensional minisuperspace ${ }^{2}$ (but in different coordinates).

The conservation laws are

$$
\begin{gathered}
I_{\varphi}(a, \dot{a}, \varphi, \dot{\varphi})=a^{2} \varphi^{2} \dot{a}-4 a^{3} \varphi \dot{\varphi}, \\
I_{\Phi}(a, \dot{a}, \Phi, \dot{\Phi})=a^{2}(\lambda \dot{a}-a \dot{\Phi}), \\
I_{\sigma}(a, \dot{a}, \sigma, \dot{\sigma})=2 a^{2} \sigma(\mu-2) \dot{a}+a^{3}(\mu+1) \dot{\sigma}
\end{gathered}
$$

while the corresponding generators of the conservation laws are

$$
X_{\varphi}=a \partial_{a}-\frac{3}{2} \varphi \partial_{\varphi}, X_{\Phi}=a \partial_{a}+\frac{6}{\lambda} \partial_{\Phi}, X_{\sigma}=\frac{(\mu+1)}{6} a \partial_{a}+\sigma \partial_{\sigma},
$$

which are Noether (point) symmetries for the Lagrangians (19), (20), and (21) respectively.

However, functions $I_{\Phi}, I_{\sigma}$ are "weak" conservation laws in the sense that someone has to impose the constraint condition $\mathcal{H}=0$, in order $\frac{d I}{d t}=0$, and that is because it is held $\frac{d I_{\Phi}}{d t}=2 \mathcal{H}_{\Phi}$ and $\frac{d I_{\sigma}}{d t}=2 \mathcal{H}_{\sigma}$. Hence, vector fields $X_{\Phi}$, and $X_{\sigma}$ are Homothetic symmetries for the minisuperspace of Lagrangians $L_{\Phi}$, and $L_{\sigma}$, and only $X_{\varphi}$ is a Killing symmetry, for $L_{\varphi}$, which is a necessity condition in order Gasperini-Veneziano duality transformation to exist. Therefore, we can say that duality invariance is lost under conformal transformations and the power-law $f(R)$-gravity does not admit any duality symmetry, for arbitrary value $\mu$, such that $\mu \neq 0,1$.

\subsection{Classical mechanical analogue}

In order to find the classical mechanical analogue of the Gasperini-Veneziano duality transformation we perform the change of variables

$$
a=u^{\frac{2}{7}-\frac{\sqrt{15}}{21}} v^{\frac{2}{7}+\frac{\sqrt{15}}{21}}, \varphi=u^{\frac{1}{14}+\frac{\sqrt{15}}{14}} v^{\frac{1}{14}-\frac{\sqrt{15}}{14}}
$$

in (19) where $u=x+y$ and $v=x-y$. Because that is a coordinate transformation the new Lagrangian is equivalent with the original without imposing any constraint [35]. The resulting Lagrangian after the coordinate transformation (26) is

$$
L_{\varphi}(x, \dot{x}, y, \dot{y})=\frac{1}{2}\left(\dot{x}^{2}-\dot{y}^{2}\right)+\bar{\Lambda}\left(x^{2}-y^{2}\right)
$$

\footnotetext{
${ }^{1}$ It is important to mention that conformal equivalent theories are not necessary and physical equivalent, for a discussion see [34].

2 For more details on how those conservation laws are constructed and related, we refer the reader to [29-33] and references therein.
} 
which describes the hyperbolic "oscillator" in a two-dimensional Riemannian manifold of Lorentzian signature. The field equations are well known that are maximally symmetric [36-39] and the rotation symmetry of the $M^{2}$ space provides the discrete dual symmetry $x \rightarrow y, y \rightarrow x$, which in the original coordinates of $\{a, \phi\}$ becomes the GasperiniVeneziano duality symmetry. That is an alternative way to prove that the original of the Gasperini-Veneziano duality transformation is the $O(d, d)$ symmetry.

\section{CONCLUSIONS}

In this letter, we studied the Gasperini-Veneziano duality symmetry for the Dilaton field for conformal equivalent theories. We found that duality symmetry does not survive under conformal transformations and we show that while all the conformal equivalent theories admit a conservation law of the same origin, only in the frame of the Dilaton field the conservation law follows from an isometry of the Lagrangian. Furthermore, we wrote the Lagrangian of the Dilaton field in it's classical mechanical analogue of the two-dimensional hyperbolic "oscillator" where the duality symmetry becomes the rotation symmetry, the so-called $O(d, d)$ symmetry.

Finally, in the context of $f(R)$-gravity, we show that there is not any duality symmetry for the power-law theory while the analysis which we presented in this work can be applied in extendent theories to determine discrete symmetries.

\section{Acknowledgments}

AP was financially supported by FONDECYT grants 3160121 and want to thank Guy Consolmagno, S.J. and the members of the Vatican Observatory (Specola Vaticana) for the invitation and the hospitality provided while this work was carried out.

[1] V. Faraoni, Cosmology in Scalar-Tensor Gravity, Fundamental Theories of Physics vol. 139, (Kluwer Academic Press: Netherlands, 2004).

[2] T. Clifton, P.G. Ferreira, A. Padilla and C. Skordis, Phys. Rep. 513, 1 (2012)

[3] S. Nojiri, S.D. Odintsov and M. Sasaki, Phys. Rev. D 71, 123509 (2005)

[4] K. Bamba, C.-Q. Geng, C.-C. Lee and L.-W. Luo, JCAP 01, 021 (2011)

[5] G. Kofinas and M. Tsoukalas, EPJC 76, 686 (2016)

[6] S. Nojiri and S.D. Odintsov, Phys. Rep. 50559 (2011)

[7] T.H. Buscher, Phys. Lett. B 194, 59 (1987)

[8] T.H. Buscher, Phys. Lett. B 201, 466 (1988)

[9] R. Brustein, M. Gasperini and G. Veneziano, Phys. Lett. B 431, 277 (1998)

[10] E. Alvarez, L. Alvarez-Gaume and Y. Lozano, Nucl. Phys. Proc. Suppl. 41, 1 (1995)

[11] A. Giveon, M. Porrati and E. Rabinovici, Phys. Rept. 244, 77 (1994)

[12] L. Anguelova, Proc. Math. Stat. 191, 285 (2016)

[13] Y.-G. Shen and D.-Ming Chen, Gen. Relativ. Gravit. 32, 353 (2000)

[14] Y.-F. Cai, H. Li, Y.-S. Piao and X. Zhang, Phys. Lett. B 646, 141 (2007)

[15] A.A. Kehagias and A. Lukas, Nucl. Phys. B 477, 549 (1996)

[16] K.A. Meissner and G. Veneziano, Mod. Phys. Lett. A 6, 3397 (1991)

[17] K.A. Meissner and G. Veneziano, Phys. Lett. B 267, 564 (1991)

[18] M. Gasperini and G. Veneziano, Astropart. Phys 1, 317 (1993)

[19] M. Gasperini and G. Veneziano, Phys. Rept. 373, 1 (2003)

[20] M. Gasperini, J. Maharana and G. Veneziano, Nucl. Phys. B 472, 349 (1996)

[21] S. Capozziello, G. Gionti, S.J. and D. Vierneri, JCAP 1601, 015 (2016)

[22] A. Paliathanasis and S. Capozziello, Mod. Phys. Lett. A 31, 1650183 (2016)

[23] S.W. Hawking and G.F.R. Ellis, The Large Scale Structure of Space-Time, Cambridge University Press, Cambridge, New York (1975)

[24] H.A. Buchdahl, Mon. Not. Roy. Astron. Soc. 150 (1970) 1

[25] J. O'Hanlon, Phys. Rev. Lett. 29137 (1972)

[26] T.P. Sotiriou and V. Faraoni, Rev. Mod. Phys. 82, 451 (2010)

[27] J. Klusoň, Class. Quant. Grav. 28, 125025 (2011)

[28] G.W. Horndeski, Int. J. Ther. Phys. 10, 363 (1974)

[29] S. Basilakos, M. Tsamparlis and S. Basilakos, Phys. Rev. D 83, 103512 (2011)

[30] A. Paliathanasis, M. Tsamparlis and S. Basilakos, Phys. Rev. D 84, 123514 (2011) 
[31] A. Paliathanasis, M. Tsamparlis, S. Basilakos and J.D. Barrow, Phys. Rev. D 93, 043528 (2016)

[32] T. Christodoulakis, N. Dimakis and P.A. Terzis, J. Geom. Phys. 71, 127 (2013)

[33] P.A. Terzis, N. Dimakis and T. Christodoulakis, Phys. Rev. D 90, 123543 (2014)

[34] V. Faraoni and E. Gunzig, Int. J. Theor. Phys. 38, 217 (1999)

[35] V.I. Arnold, Mathematical Methods of Classical Mechanics, Graduate texts in mathematics, Springer-Verlag New York, Second edition (1980)

[36] M. Lutzky, J. Phys. A: Math. Gen. 11, 249 (1978)

[37] T. J. Gordon, J. Phys. A: Math. Gen. 19, 183 (1986)

[38] P.G.L. Leach, J. Math. Phys. 21, 300 (1980)

[39] G. E. Prince and C. J. Eliezer, J. Phys. A: Math. Gen. 13, 815 (1980) 